PROCEEDINGS OF THE

AMERICAN MATHEMATICAL SOCIETY

Volume 126, Number 9, September 1998, Pages 2819-2825

S 0002-9939(98)04359-7

\title{
NOTES ON HOMOLOGY COBORDISMS OF PLUMBED HOMOLOGY 3-SPHERES
}

\author{
NIKOLAI SAVELIEV
}

(Communicated by Ronald A. Fintushel)

\begin{abstract}
The gauge-theoretical invariants of Donaldson and Seiberg-Witten are used to detect some infinite order elements in the homology cobordism group of integral homology 3 -spheres.
\end{abstract}

This paper is concerned with the homology cobordism group $\Theta_{\mathbb{Z}}^{3}$ of oriented integral homology 3-spheres. We use S. Donaldson's [D] and M. Furuta's [F] (see also [A]) theorems and the $\bar{\mu}$-invariant introduced by W. Neumann $[\mathrm{N}]$ and L. Siebenmann $[\mathrm{S}]$ to prove the following two theorems.

Theorem 1. Let a homology sphere $\Sigma$ be the link of an algebraic singularity. If $\Sigma$ is homology cobordant to zero, then $\bar{\mu}(\Sigma) \geq 0$.

All Seifert fibered homology spheres are the links of algebraic singularities. Therefore, one can apply this theorem to recover some of the results of R. Fintushel and R. Stern [FS] on homology cobordisms of Seifert fibered homology spheres. For example, it easily follows that for any relatively prime positive integers $p$ and $q$, Seifert fibered homology spheres $\Sigma(p, q, p q k-1), k \geq 1$, have infinite order in the group $\Theta_{\mathbb{Z}}^{3}$. Theorem 1 implies some new results as well; see Section 2.

Theorem 2. Let $\Sigma$ be a plumbed homology sphere and assume that $\bar{\mu}(\Sigma) \neq 0$. If $\Sigma$ bounds a plumbed 4-manifold with even intersection form of rank not exceeding $10|\bar{\mu}(\Sigma)|$, then $\Sigma$ has infinite order in the group $\Theta_{\mathbb{Z}}^{3}$.

One can easily see $[\mathrm{NR}]$ that any Seifert sphere $\Sigma\left(a_{1}, \ldots, a_{n}\right)$ with one of the $a_{i}$ 's even bounds an (essentially unique) plumbed manifold with even intersection form. This intersection form is very often, though not always, of the desired rank not exceeding $10|\bar{\mu}|$. Moreover, in many cases the rank can be reduced with the help of Kirby calculus without changing $\bar{\mu}$ so that the conclusion of Theorem 2 still holds. Such an approach leads in particular to the following result [S1].

Corollary 1. For any relatively prime integers $p, q \geq 2$ and any odd integer $k \geq 1$ the Seifert fibered homology sphere $\Sigma(p, q, p q k+1)$ has infinite order in the group $\Theta_{\mathbb{Z}}^{3}$.

This result cannot be extended to $\Sigma(p, q, p q k+1)$ with even $k$, because, for instance, $\Sigma(2,3,13)$ is known to bound a contractible manifold.

Received by the editors November 19, 1996 and, in revised form, February 11, 1997.

1991 Mathematics Subject Classification. Primary 57M25, 57R80.

Key words and phrases. Homology 3-sphere, homology cobordism, gauge theory.

(C)1998 American Mathematical Society 
It is worth mentioning that in fact $\bar{\mu}(\Sigma)$ vanishes for all known examples of plumbed homology spheres $\Sigma$ homology cobordant to zero. In [N], W. Neumann conjectured that $\bar{\mu}$ is a homology cobordism invariant. The results of this paper give further evidence which weighs for a positive solution to this conjecture.

\section{Definition AND BASIC PROPERTIES OF THE $\bar{\mu}$-INVARIANT}

We recall the definition of the invariant $\bar{\mu}$ by W. Neumann $[\mathrm{N}]$. Note that our definition differs from the original one by a factor of $1 / 8$.

Let $\Gamma$ be a connected plumbing graph, that is, a connected graph with no cycles, each of whose vertices carries an integer weight $e_{i}, i=1, \ldots, s$. The matrix $A(\Gamma)=\left(a_{i j}\right)_{i, j=1, \ldots, s}$ with the entries

$$
a_{i j}= \begin{cases}e_{i}, & \text { if } i=j, \\ 1, & \text { if } i \text { is connected to } j \text { by an edge, } \\ 0, & \text { otherwise, }\end{cases}
$$

is the intersection matrix of the 4-dimensional manifold $P(\Gamma)$ obtained by plumbing $D^{2}$-bundles over 2 -spheres according to $\Gamma$. This manifold is simply connected.

Disconnected graphs are also allowed. Namely, if $\Gamma=\Gamma_{0}+\Gamma_{1}$ is a disjoint union of $\Gamma_{0}$ and $\Gamma_{1}$, then $P(\Gamma)$ is the boundary connected sum $P\left(\Gamma_{0}\right) \llbracket P\left(\Gamma_{1}\right)$.

If $\Gamma$ is a plumbing graph as above, then $M(\Gamma)=\partial P(\Gamma)$ is an integral homology sphere if and only if $\operatorname{det} A(\Gamma)= \pm 1$. For example, all Seifert fibered homology spheres $\Sigma\left(a_{1}, \ldots, a_{n}\right)$ are of the form $\partial P(\Gamma)$ where $\Gamma$ is a star-shaped graph; see $[\mathrm{NR}]$.

If $M(\Gamma)$ is a homology sphere, there is a unique homology class $w \in H_{2}(P(\Gamma) ; \mathbb{Z})$ satisfying the following two conditions. First, $w$ is characteristic, that is (dot represents intersection number)

$$
w . x \equiv x . x \quad \bmod 2 \quad \text { for all } x \in H_{2}(P(\Gamma) ; \mathbb{Z}),
$$

and second, all coordinates of $w$ are either 0 or 1 in the natural basis of $H_{2}(P(\Gamma) ; \mathbb{Z})$. We call $w$ the integral $W u$ class for $P(\Gamma)$. It follows from $[\mathrm{N}]$ that the integer sign $P(\Gamma)-w \cdot w$ depends only on $M(\Gamma)$ and not on $\Gamma$. This integer is divisible by 8 , so one can define the Neumann-Siebenmann invariant by the formula

$$
\bar{\mu}(M(\Gamma))=\frac{1}{8}(\operatorname{sign} P(\Gamma)-w \cdot w) .
$$

It is also easily seen that (1) implies that no two coordinates of $w$ corresponding to adjacent vertices in $\Gamma$ can both be 1 , so it follows that $w$ is spherical, and the modulo 2 reduction of the $\bar{\mu}$-invariant is the usual Rohlin invariant $\mu$; see [NR].

\section{The $\bar{\mu}$-INVARIANT OF ALgEBRAIC LINKS}

The plumbed homology spheres have been classified in [EN]. In particular, it has been shown that a plumbed homology sphere $\Sigma$ is an algebraic link if and only if there exists a plumbing graph $\Gamma$ such that the intersection form of the manifold $P(\Gamma)$ with $\Sigma=\partial P(\Gamma)$ is negative definite. The simplest case is the Seifert fibered case: any Seifert fibered homology sphere $\Sigma\left(a_{1}, \ldots, a_{n}\right)$ is the link of the singularity of $f^{-1}(0)$, where $f: \mathbb{C}^{n} \rightarrow \mathbb{C}^{n-2}$ is a map of the form

$$
f\left(z_{1}, \ldots, z_{n}\right)=\left(\sum_{k=1}^{n} b_{1, k} z_{k}^{a_{k}}, \ldots, \sum_{k=1}^{n} b_{n-2, k} z_{k}^{a_{k}}\right)
$$


with sufficiently general coefficient matrix $\left(b_{i, j}\right)$; see $[\mathrm{NR}]$. For instance,

$$
\Sigma(p, q, r)=\left\{z \in \mathbb{C}^{3} \mid\|z\|=\varepsilon \text { and } z_{1}^{p}+z_{2}^{q}+z_{3}^{r}=0\right\}
$$

for $\varepsilon>0$ small enough.

The next simplest case is the following: if $p, q, r$ are pairwise relatively prime integers, as are $p^{\prime}, q^{\prime}, r^{\prime}$, then the homology sphere $\Sigma$ obtained by splicing $\Sigma(p, q, r)$ and $\Sigma\left(p^{\prime}, q^{\prime}, r^{\prime}\right)$ along the singular fibers of degrees $r$ and $r^{\prime}$ is the link of singularity if and only if $r r^{\prime}>p p^{\prime} q q^{\prime}$; see [NW], $\S 4$.

Theorem 1. Let a homology sphere $\Sigma$ be the link of an algebraic singularity. If $\Sigma$ is homology cobordant to zero, then $\bar{\mu}(\Sigma) \geq 0$.

Proof. Since $\Sigma$ is an algebraic link, one may assume that $\Sigma$ is the boundary of a plumbed 4-manifold $P(\Gamma)$ whose intersection form is negative definite. Suppose that $\Sigma$ bounds a smooth homology ball $M$. Let us consider the manifold $W=$ $P(\Gamma) \cup_{\Sigma}(-M)$. This is a smooth closed oriented manifold whose intersection form is naturally isomorphic to the intersection form of $P(\Gamma)$, in particular, is negative definite. By S. Donaldson's Theorem 1 from [D], this form is diagonalizable over the integers.

We use this fact to evaluate $\bar{\mu}(\Sigma)$. Obviously, $\operatorname{sign} P(\Gamma)=-s$, where $s$ is the number of vertices in the graph $\Gamma$, so we only need to find the Wu class $w$. In the standard basis associated with the plumbing, the matrix $A$ of the intersection form of $P(\Gamma)$ takes the form $A=U^{t}(-E) U$, where $U \in \mathrm{SL}_{s}(\mathbb{Z})$ and $E$ is the identity matrix. The defining relation (1) translates to

$$
w^{t} U^{t}(-E) U x \equiv x^{t} U^{t}(-E) U x \quad \bmod 2 \quad \text { for all } x \in H_{2}(P(\Gamma) ; \mathbb{Z}),
$$

or, equivalently,

$$
(U w)^{t}(-E) y \equiv y^{t}(-E) y \quad \bmod 2 \quad \text { for all } y \in H_{2}(P(\Gamma) ; \mathbb{Z}) .
$$

Therefore, $U w$ is characteristic for $-E$, in particular, all the coordinates of $U w$ are odd. Now, we have

$$
w \cdot w=w^{t} U^{t}(-E) U w=-(U w)^{t}(U w),
$$

which is equal to minus the square of the Euclidean length of the vector $U w$. Since all the coordinates of $U w$ are odd, $w \cdot w \leq-s$, and therefore $\bar{\mu}(\Sigma) \geq 0$.

Corollary 2. If a plumbed homology sphere $\Sigma$ is an algebraic link and $\bar{\mu}(\Sigma)<0$, then $\Sigma$ has infinite order in the group $\Theta_{\mathbb{Z}}^{3}$.

Proof. Let $m \Sigma=\Sigma \# \ldots \# \Sigma$ ( $m$ times). Since $\bar{\mu}$ is additive with respect to connected sums, $\bar{\mu}(m \Sigma)=m \bar{\mu}(\Sigma)<0$, therefore, $m \Sigma$ is not homology cobordant to zero for any $m$.

Example. For any relatively prime integers $p, q>0$, one can easily see that $\bar{\mu}(\Sigma(p, q, p q-1))<0$. Therefore, all these homology spheres are of infinite order in $\Theta_{\mathbb{Z}}^{3}$. In particular, the Poincaré homology sphere $\Sigma(2,3,5)$ is of infinite order.

Example. Homology spheres $\Sigma(p, q, p q k-1)$ have infinite order in $\Theta_{\mathbb{Z}}^{3}$ for any $k \geq 1$. If $k$ is odd, this holds since $\bar{\mu}(\Sigma(p, q, p q k-1))$ equals $\bar{\mu}(\Sigma(p, q, p q-1))$ and is therefore negative. If $k$ is even, then $\bar{\mu}(\Sigma(p, q, p q k-1))=0$, and the argument of Theorem 1 cannot be applied directly. Let $A$ be the intersection form of a negative definite plumbing $X$ bounding $\Sigma(p, q, p q k-1), k$ even. We perform a (-1)-surgery 
on the singular fiber of degree $p q k-1$ to get $\Sigma(p, q, p q(k+1)-1)$. The union of the cobordism $X$ with the trace of this surgery is a plumbed manifold with the intersection form $A \oplus(-1)$. Now, if $\Sigma(p, q, p q k-1)$ bounded an acyclic manifold, $A$ would be diagonalizable, and so would be $A \oplus(-1)$. This contradicts the fact that $\bar{\mu}(\Sigma(p, q, p q(k+1)-1))<0$. A similar argument shows that none of the multiples of $\Sigma(p, q, p q k-1)$ is homology cobordant to zero.

Many Seifert homology spheres $\Sigma\left(a_{1}, \ldots, a_{n}\right)$ having infinite order in the group $\Theta_{\mathbb{Z}}^{3}$ by Corollary 2 can also be detected by the $R$-invariant of R. Fintushel and R. Stern,

$$
R\left(a_{1}, \ldots, a_{n}\right)=\frac{2}{a}-3+n+\sum_{i=1}^{n} \frac{2}{a_{i}} \sum_{k=1}^{a_{i}-1} \cot \left(\frac{\pi a k}{a_{i}^{2}}\right) \cot \left(\frac{\pi k}{a_{i}}\right) \sin ^{2}\left(\frac{\pi k}{a_{i}}\right),
$$

where $a=a_{1} \cdot \ldots \cdot a_{n}$. A theorem in [FS] implies that if $\Sigma\left(a_{1}, \ldots, a_{n}\right)$ bounds a homology ball, then $R\left(a_{1}, \ldots, a_{n}\right)<0$. There are however Seifert fibered homology spheres which are not homology cobordant to zero and which can be detected by $\bar{\mu}$ and not by $R$, and vice versa.

Example. Both Seifert spheres $\Sigma(2,11,19)$ and $\Sigma(3,5,7)$ are not homology cobordant to zero. As to the former one, this follows from the fact that $\bar{\mu}(\Sigma(2,11,19))=$ -1 is negative (though $R(2,11,19)=-1$ ); the latter one has $\bar{\mu}(\Sigma(3,5,7))=0$ and the result follows from $R(3,5,7)=1$.

The $\bar{\mu}$-invariant also detects some plumbed homology spheres which have infinite order in $\Theta_{\mathbb{Z}}^{3}$ but are not Seifert fibered.

Example. Let $\Sigma$ be the splice of $\Sigma(4,7,9)$ and $\Sigma(2,3,25)$ along the singular fibers of degrees 9 and 25 . This manifold is an algebraic link. By using the additivity of the $\bar{\mu}$-invariant proven in $[\mathrm{Du}]$ and $[\mathrm{S} 2]$, we find that

$$
\bar{\mu}(\Sigma)=\bar{\mu}(\Sigma(4,7,9))+\bar{\mu}(\Sigma(2,3,25))=-2+0<0 .
$$

Therefore, $\Sigma$ has infinite order in $\Theta_{\mathbb{Z}}^{3}$ (although the Rohlin invariant $\mu(\Sigma)$ equals 0 modulo 2 ).

\section{More CONSTRAints From EVEn PLUMBing}

In 1995, M. Furuta [F] used the Seiberg-Witten invariants to prove the so-called 10/8-conjecture, which says that if $A$ is the intersection form of a smooth closed spin 4-manifold, then $\operatorname{rank} A /|\operatorname{sign} A|>10 / 8$. In this section we use this result to prove the following theorem.

Theorem 2. Let $\Sigma$ be a plumbed homology sphere and assume that $\bar{\mu}(\Sigma) \neq 0$. If $\Sigma$ bounds a plumbed 4-manifold with even intersection form of rank not exceeding $10|\bar{\mu}(\Sigma)|$, then $\Sigma$ has infinite order in the group $\Theta_{\mathbb{Z}}^{3}$.

Proof. Let $P(\Gamma)$ be a plumbed manifold with boundary $\partial P(\Gamma)=\Sigma$, such that its intersection form $A=A(\Gamma)$ is even and has rank not exceeding $10|\bar{\mu}(\Sigma)|$. Since $A$ is even, its $\mathrm{Wu}$ class $w$ vanishes. Therefore, $8 \cdot \bar{\mu}(\Sigma)=\operatorname{sign} A$, and moreover $A$ is isomorphic over the integers to $|\bar{\mu}(\Sigma)| \cdot E_{8} \oplus b \cdot H$. Since $\operatorname{rank}(A) \leq 10|\bar{\mu}(\Sigma)|$, we get that $\operatorname{rank} A /|\operatorname{sign} A| \leq 10 / 8$. 
Now, suppose that $\Sigma$ bounds a smooth homology ball $M$, and consider the manifold $W=P(\Gamma) \cup_{\Sigma}(-M)$. This is a smooth closed oriented spin 4-manifold whose intersection form $Q$ is isomorphic to $|\bar{\mu}(\Sigma)| \cdot E_{8} \oplus b \cdot H$. Since $\operatorname{rank} Q /|\operatorname{sign} Q| \leq$ $10 / 8$, we get a contradiction with the $10 / 8$ conjecture.

The argument can be repeated with $\Sigma$ replaced by any of its multiples, which proves the theorem.

For a complete proof of Corollary 1 formulated in the introduction we refer the reader to [S1], and only prove it here in the simplest case $p=2$. If $q \equiv 3 \bmod 4$, then $\Sigma(2, q, 2 q k+1), k$ odd, is the boundary of the 4 -manifold obtained by plumbing according to the following diagram:

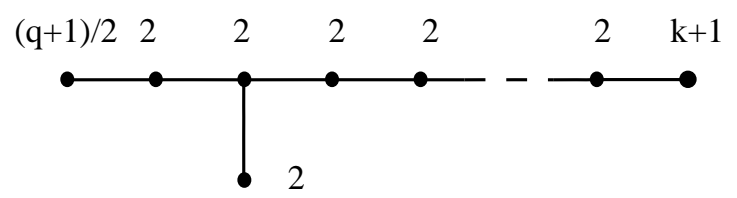

The intersection form of this manifold is isomorphic to $\frac{q+1}{4} \cdot E_{8} \oplus H$. If $q \equiv 1$ $\bmod 4$, the homology sphere $\Sigma(2, q, 2 q k+1)$ bounds plumbing

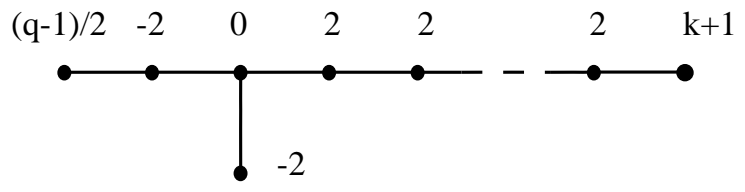

with intersection form $\frac{q-1}{4} \cdot E_{8} \oplus 3 \cdot H$. To deal with this case, we need to reduce the rank by surgering out two "hyperbolics" $H$. This can be done as follows. First, redraw the plumbing diagram:

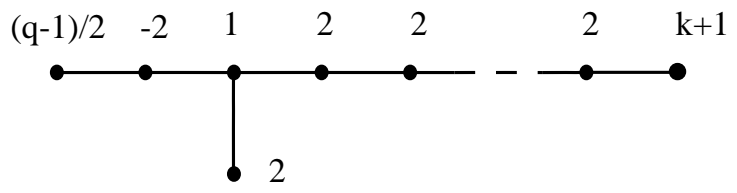

After two obvious blow-downs we get the manifold in Figure 1 with the intersection form $\frac{q-1}{4} \cdot E_{8} \oplus 2 \cdot H$. An equivalent link description is shown in Figure 2 . After two more blow-downs we get rid of another "hyperbolic" $H$. The final link description is shown in Figure 3.

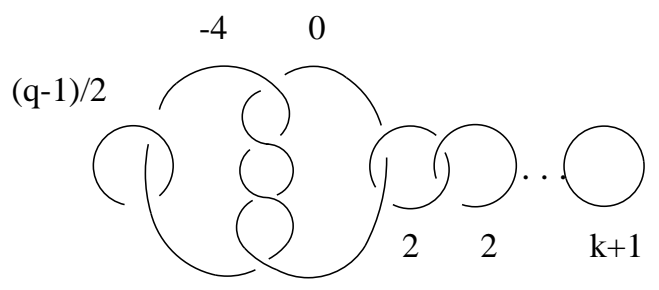

FIGURE 1 


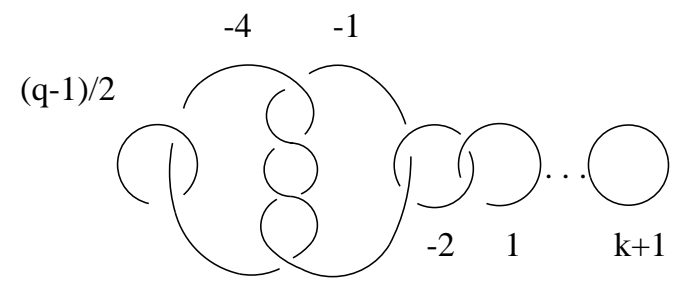

FiguRE 2

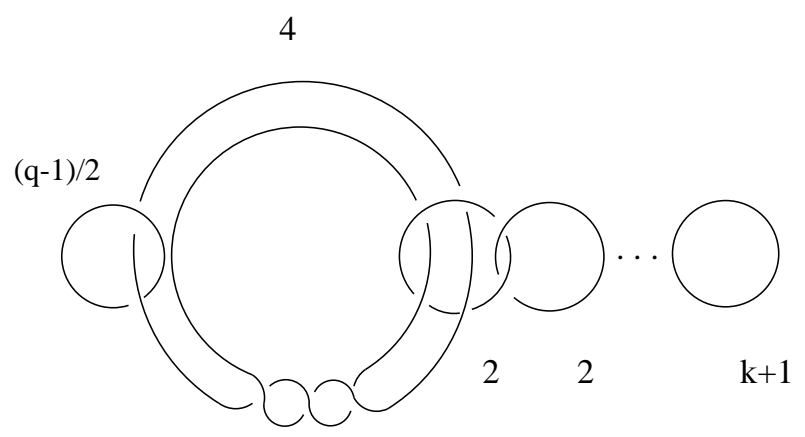

FiguRE 3

The corresponding intersection form is isomorphic to $\frac{q-1}{4} \cdot E_{8} \oplus H$, and an argument similar to that in Theorem 2 can be used to show that $\Sigma(2, q, 2 q k+1), k$ odd, has infinite order in $\Theta_{\mathbb{Z}}^{3}$.

Example. In addition to those listed in Corollary 1, there are of course many homology spheres having infinite order in $\Theta_{\mathbb{Z}}^{3}$ due to Theorem 2. For example, $\Sigma(8,13,21)$ is of this sort because it has $\bar{\mu}$-invariant equal 4 and can be obtained by plumbing on an even weighted graph of rank 34 .

\section{REFERENCES}

[A] S. Akbulut, Lectures on Seiberg-Witten Invariants, Turkish J. Math. 20 (1996), 95-118. MR 97g:57038

[D] S. Donaldson, The orientation of Yang-Mills moduli spaces and 4-manifold topology, J. Diff. Geom. 26 (1987), 397-428. MR 88j:57020

[Du] N. Duchon, Involutions of plumbed manifolds, Ph.D. Thesis, University of Maryland, College Park, 1982.

[EN] D. Eisenbud, W. Neumann, Three-dimensional Link Theory and Invariants of Plane Curve Singularities, Annals of Math. Studies 110, Princeton, 1985. MR 87g:57007

[FS] R. Fintushel, R. Stern, Pseudofree orbifolds, Ann. Math. 122 (1985), 335-364. MR 87a:57027

[F] M. Furuta, Monopole equation and the 11/8 conjecture, Preprint.

$[\mathrm{N}] \quad$ W. Neumann, An invariant of plumbed homology spheres, Lecture Notes in Math. 788, Springer-Verlag, Berlin and New-York, 1980, 125-144. MR 82j:57033

[NR] W. Neumann, F. Raymond, Seifert manifolds, plumbing, $\mu$-invariant and orientation reversing maps, Lecture Notes in Math. 664, Springer-Verlag, Berlin and New-York, 1978, 163-196. MR 80e:57008

[NW] W. Neumann, J. Wahl, Casson invariant of links of singularities, Comm. Math. Helv. 65 (1990), 58-78. MR 91c:57022

[S1] N. Saveliev, Dehn surgery along torus knots, Topology and Its Appl. (to appear). 
[S2] N. Saveliev, Floer homology and 3-manifold invariants, Ph.D. Thesis, University of Oklahoma, Norman, 1995.

[S] L. Siebenmann, On vanishing of the Rohlin invariant and nonfinitely amphicheiral homology 3-spheres, Lecture Notes in Math. 788, Springer-Verlag, Berlin and New-York, 1980, 172-222. MR 81k:57011

Department of Mathematics, University of Michigan, Ann Arbor, Michigan 48109

E-mail address: saveliev@math.lsa.umich.edu 